\title{
The detectability of sea-ice leads in satellite data as a function of atmospheric conditions and measurement scale
}

\author{
J. Key, R. Stone, J. Maslanik and E. Elleffsen \\ Division of Cryospheric and Polar Processes, Cooperative Institute for Research in Environmental Sciences, University of Colorado, \\ Boulder, CO 80309-0449, U.S.A.
}

\begin{abstract}
The release of heat from sea-ice leads is an important component of the heat budget in the Arctic, but the impact of leads on regional scale climate is difficult to assess without information on their distribution in both space and time. Remote sensing of leads using satellite data, specifically AVHRR thermal and Landsat visible imagery, is examined with respect to one lead parameter: lead width. Atmospheric effects are illustrated through the concept of thermal contrast transmittance, where the brightness temperature contrast between leads of various ice thicknesses and the surrounding multi-year ice is simulated using a radiative transfer model. Calculations are made as a function of aerosol, ice crystal precipitation, and cirrus cloud optical depths. For example, at ice crystal optical depths of more than about 1.5 under mean January conditions in the central Arctic, the brightness temperature differences between $2 \mathrm{~m}$ and $5 \mathrm{~cm}$ thick ice are similar to the ice temperature variability so that there would be insufficient contrast to distinguish a lead from the surrounding ice. The geometrical aspects of the sensor are also simulated by degrading Landsat data so that the effect of sensor field-of-view on retrieved lead width statistics can be assessed. Large leads tend to "grow" with increased pixel size while small leads disappear. Changes in lead width and orientation distributions can readily be seen.
\end{abstract}

\section{INTRODUCTION}

Sea-ice leads (linear openings in the ice pack) are an important component in local scale heat exchange in the Arctic, providing a significant source of heat and moisture to the atmosphere. Unfortunately, their impact on regional scale climate is difficult to assess without more information on their frequency of occurrence in both space and time. The use of satellite data, and in particular the thermal channels of the Advanced Very High Resolution Radiometer (AVHRR), for the detection and mapping of leads has been suggested, but its utility is questionable because of its relatively low resolution $(1.1 \mathrm{~km}$ at nadir $)$ and the influence of the intervening atmosphere on upwelling radiation. It is necessary, therefore, to understand how sensor characteristics, atmospheric properties, and surface conditions influence the detection and interpretation of sea-ice leads in AVHRR and other satellite data. We seek to determine the sources and magnitudes of errors inherent in the measurements and how lead statistics change with the different spatial resolutions of existing and future sensors.

Specifically, we address the following issues. What is actually being measured by these sensors? To what extent do surface, atmosphere, and sensor factors affect lead observations and image interpretations? How does lead detection depend on ice thickness as a function of sensor response, surface temperatures, and atmospheric condi- tions? Since the temperature contrast between open water and ice provides a means to map leads using thermal imagery, to what degree does this contrast affect the apparent width of a lead and our ability to detect it? How do atmospheric, boundary layer, and solar zenith angle effects combine with surface conditions and viewing geometry to determine the thresholds of lead detection?

To address these questions our approach includes both modeling and empirical studies. Radiative transfer modeling is done for the purpose of simulating the satellite sensor response under a variety of atmospheric and surface conditions. The empirical studies include a comparison of lead statistics determined in imagery of varying scales (e.g. AVHRR, Landsat and OLS data) and the use of lead statistics derived from low resolution imagery to estimate characteristics of the distributions which could be obtained using high resolution imagery. In this paper we discuss both the empirical and modeling studies with respect to lead width, which is defined as the distance across a lead along a line perpendicular to the local orientation of the lead.

\section{THERMAL CONTRAST BETWEEN LEADS AND THE SURROUNDING ICE}

Atmospheric and view angle effects are modeled under a variety of conditions to simulate sensor response in order 
to gain a better understanding of how leads might appear in the satellite data under varying surface and atmospheric conditions. With this knowledge, it may be possible to estimate the smallest resolvable lead under a given set of surface/atmospheric/sensor resolution and view angle conditions. Emphasis is on the AVHRR thermal channels (3: centered at approximately $3.7 \mu \mathrm{m}, 4: 11 \mu \mathrm{m}, 5$ : $12 \mu \mathrm{m})$.

To simulate radiances measured by the sensor, the LOWTRAN 7 (hereafter LOWTRAN) atmospheric transmittance/radiance model (Kneizys and others, 1988) is used. LOWTRAN calculates atmospheric transmittance/radiance for wave numbers ranging from 0 to $50000 \mathrm{~cm}^{-1}(0.2 \mu \mathrm{m}$ to infinity) at a resolution of $20 \mathrm{~cm}^{-1}$, and includes calculations for multiple scattered radiation. The code may be run using standard or userdefined atmospheres, several cloud models, aerosol models, and specified solar and view geometry. Our analyses are based on atmospheric temperature and humidity profiles collected by radiosonde from the Soviet ice islands RVAB (approximately $80^{\circ} \mathrm{N}, 160^{\circ} \mathrm{W}$ ) during $1979-83$ and EMIO (approximately $87^{\circ} \mathrm{N}, 175^{\circ} \mathrm{E}$ ) during 1983-1987. Generally two observations per day were collected covering a vertical range of 0 to $25 \mathrm{~km}$. Profiles that had more than 10 pressure levels were retained in the analyses. Pertinent observations include temperature and dew point depression. Results for mean January conditions are presented here.
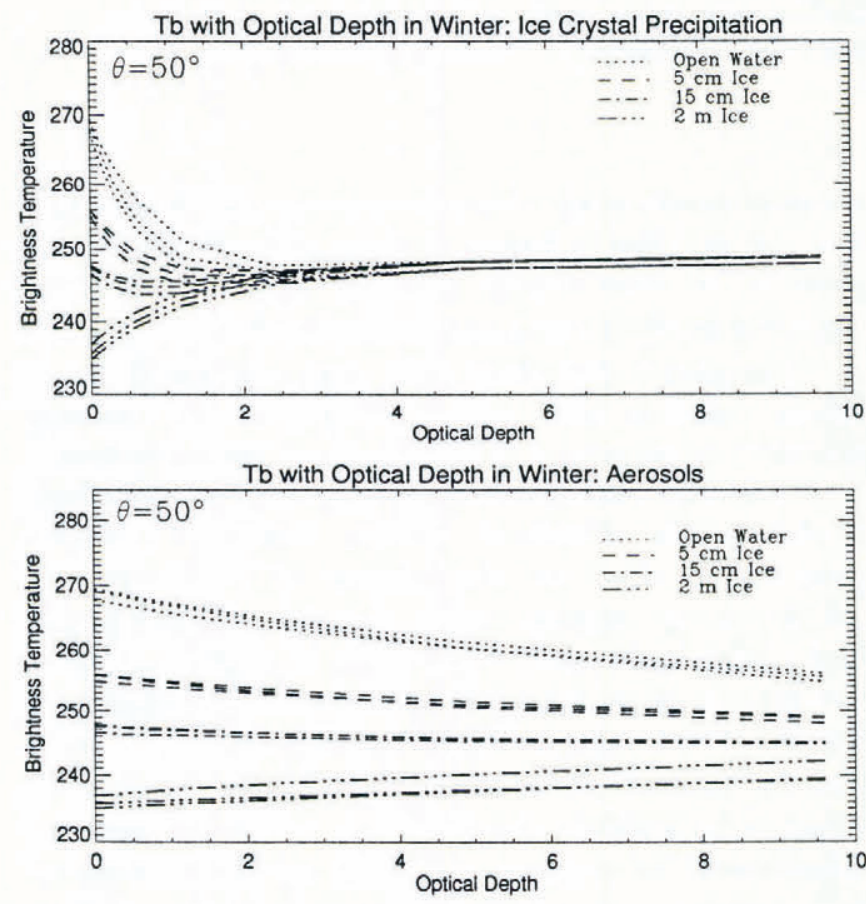

Fig. 1. The effect of a layer of ice crystal precipitation (top) and boundary layer haze (bottom) on the three AVHRR thermal channels (3, 4, 5; from top to bottom for each set of line types) at a satellite view angle of $50^{\circ}$. Brightness temperatures are given for each of four surface types (ice thicknesses: $0,5,15,200 \mathrm{~cm}$ ) as a function of $0.55 \mu \mathrm{m}$ optical depths. The tops of the ice crystal and haze layers are near the top of the inversion at a temperature of approximately $248 K$. Conditions are mean for January in the central Arctic.
The turbidity of the atmosphere due to aerosols and ice crystals can be parameterized as optical depth. LOWTRAN calculations were made over a range of $0.55 \mu \mathrm{m}$ optical depths for three prescribed surface temperatures used to characterize different lead types (open or refrozen) and a fourth to represent the "background" scene consisting of $2 \mathrm{~m}$ thick ice. These temperatures were estimated using a surface energy balance approach (Maykut, 1982) and characterized open leads $(271 \mathrm{~K})$, leads covered by $5 \mathrm{~cm}$ thick ice $(257 \mathrm{~K})$ and leads covered by $15 \mathrm{~cm}$ thick ice $(248 \mathrm{~K})$. The $2 \mathrm{~m}$ thick ice has a skin temperature of $235 \mathrm{~K}$ in all model calculations. The model was run for satellite viewing angles of $0^{\circ}$ (nadir), $20^{\circ}$ and $50^{\circ}$ (near maximum scan angle) and accounted for differences in directional emissivity at each central wavelength of the AVHRR channels. Temperature and humidity profiles were based on the mean January ice island soundings discussed above. All simulated radiances were converted to brightness temperatures for ease of analysis and intercomparisons.

Examples of the results are shown in Figure 1 for two commonly observed conditions in the Arctic: ice crystal precipitation and haze (aerosols) in the boundary layer. The upper plot in Figure 1 gives results for a $50^{\circ}$ viewing angle - where brightness temperature differences are less due to the increased path length — showing the behavior of channel brightness temperatures for simulations assuming each of the surface temperatures defined above. A hypothetical cirrus cloud of varying optical depth was inserted in the lowest $1 \mathrm{~km}$ of the atmosphere to simulate the effects of "ice crystal precipitation" which is a common feature affecting the radiative balance of the lower troposphere in the Arctic. Regardless of the underlying surface type the simulated brightness temperatures rapidly converge to the $248 \mathrm{~K}$ black-body radiating temperature of the "cloud top" level as optical depth increases. Note that because all three channels are centered in window regions of the spectrum, surface temperatures are well simulated at zero optical depths. Maximum brightness temperature differences for a given surface type exist between the channels at around optical depth 0.5 to 0.8 . If satellite radiances can be measured accurately, these differences may be used to delineate leads when optically thin ice crystal precipitation is present in the boundary layer. However, brightness temperature differences and contrast between surface types is rapidly diminished as the optical depth increases beyond about 1.0, making it impossible to detect the underlying leads. Optical depths of greater than 1.0 are not at all unexpected as optical depths of up to 20 have been reported for ice crystal precipitation (Curry and others, 1990).

The lower plot in Figure 1 shows the results assuming that an aerosol layer exists from the surface to a altitude of $2 \mathrm{~km}$ (the boundary layer aerosol model defined in LOWTRAN) over the same range of optical depths. During the daytime, multiple scattered solar radiation adds significantly to the upwelling radiances in channel 3 as optical depths of the haze layer increase (not shown). Conversely, the infrared channels do not show any multiple scattering effects. In the haze simulations the brightness temperature differences between channel pairs 

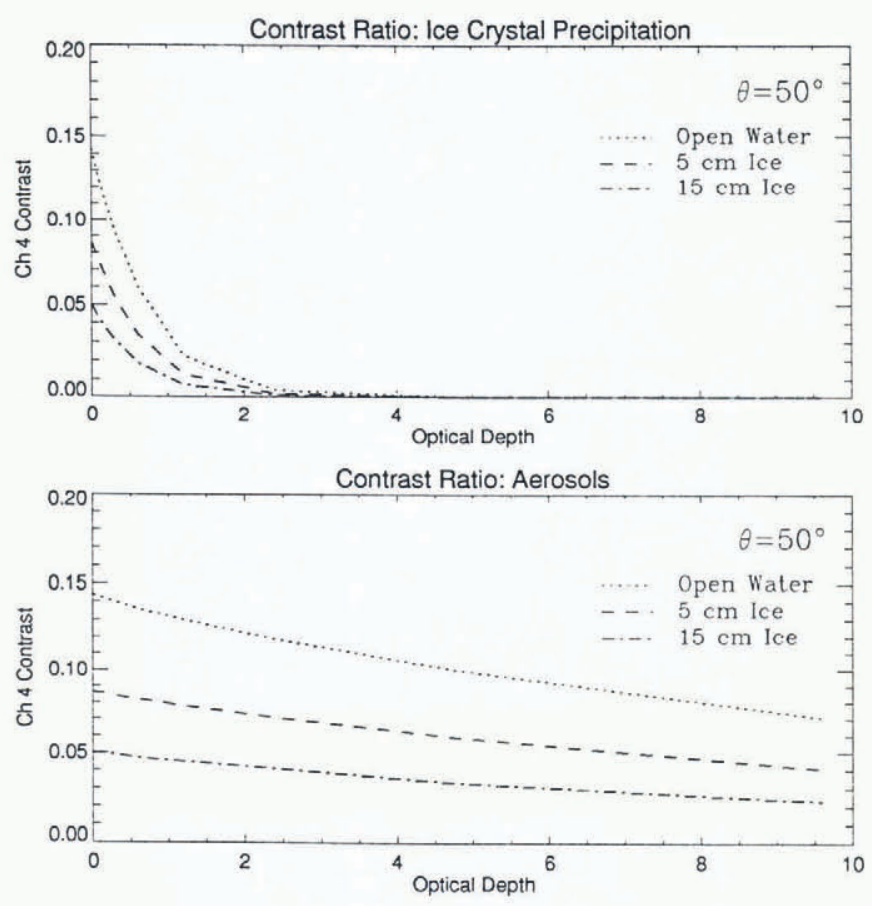

Fig. 2. Normalized AVHRR channel 4 contrast between an open lead and ice of two thicknesses for ice crystal precipitation (top) and boundary layer haze (bottom). Conditions are mean for January in the central Arctic.

are small over the entire range of optical depths for thermal radiances only, while large differences exist between channel 3, and channels 4 or 5 when solar radiation is accounted for during the day. Also, the contrast between surface types for each channel remains significant over the range of visible optical depths shown, particularly when only thermal radiances are considered.

The contrast between varying lead types and the ice pack can be expressed by the difference in simulated channel brightness temperature for a hypothetical lead temperature (e.g. open water, $5 \mathrm{~cm}$ thick ice or $15 \mathrm{~cm}$ thick ice) and the surrounding ice pack at any optical depth, normalized by the brightness temperature of the ice pack (here taken to be $2 \mathrm{~m}$ thick). Figure 2 shows the normalized contrast for channel 4 assuming the conditions illustrated in Figure 1. This relative measure of contrast may provide a means to detect leads using AVHRR data even at night, though it is apparent that, if optically thick ice crystal precipitation exists, the contrast is essentially zero making it impossible to detect leads using such a method.

Also modeled, though not illustrated here, were channel brightness temperatures, pair differences and normalized contrasts in surface types for high-level cirrus clouds at each viewing angle. Again, brightness temperatures converged at the black-body radiating temperature at cloud top for large optical depths, but the convergence was less dramatic than in the case of low-level ice crystal precipitation. Channel 3 minus channel 4 brightness temperature differences at $50^{\circ}$ viewing angle were significant in this case, reaching nearly $12 \mathrm{~K}$ over open leads at a $0.55 \mu \mathrm{m}$ optical depth of about 2.6 with the difference being less than $5 \mathrm{~K}$ over the pack ice.
Differences in these signatures when incorporated into image analysis algorithms in conjunction with ancillary information should be very useful in the detection of leads. Though the normalized contrast between surface types degrades with increasing cirrus optical depth, our simulations indicate that reasonable signatures should be detectable for a range up to about optical depth 4.0, that is, for most cirrus conditions expected to be observed in the Arctic region. Combining brightness temperature differences with contrast will improve our ability to detect leads significantly. Developing an algorithm to do so presents a challenge for the future.

\section{EMPIRICAL APPROACHES AND RESULTS}

Studies of the effect of sensor resolution on parameter retrieval have so far been empirical, dealing only with cloud fraction and a single cloud type such as cumulus, e.g. Shenk and Salomonson (1972), Wielicki and Welch (1986) or land cover classes, e.g. Woodcock and Strahler (1987), Townshend and Justice (1988). In the case of cloud fraction, real and synthetic data containing cloud fields were degraded in resolution, and the fractional coverage was observed as a function of scale. In the case of land cover classes, the variance of the image was plotted as a function of measurement scale for the purpose of determining the optimal resolution for monitoring. While all of these studies are useful, no concise statement of the relationship between fractional coverage and sensor resolution was given, so that the results are difficult to generalize to other parameters. An analytical solution to this problem is currently being investigated, but here we present results from an empirical study.

While there are advantages to comparing lead statistics derived from different types of imagery, such a study would be difficult given different acquisition times, wavelength ranges of the various sensors, and geolocation problems. To alleviate these problems, comparisons are made across image scales based on the same initial image, where the change in resolution is obtained by modeling the transfer function between the initial data and the desired resolution and then subsampling. A spatial filter that estimates the point spread function of the Landsat sensor is applied following the methodology presented in Justice and others (1989). At each degradation cycle, Gaussian random noise is added back into the image to reduce the smoothing effects of the filtering operation. Images with fields-of-view (FOV) of 160, 320, 640 and $1280 \mathrm{~m}$ were created in this manner. Data are Landsat Multispectral Scanner (MSS) band $4(0.5-0.6 \mu \mathrm{m})$ scenes of the Beaufort Sea, March 1988, with an initial FOV of $80 \mathrm{~m}$.

Leads statistics are retrieved from a lead/not-lead binary image. To obtain the binary image the fourth order trend surface is removed from original grey scale image (Eppler and Full, 1992) and an arbitrary threshold procedure is applied at each degradation cycle. Valid lead fragments are identified, where "valid" refers to a linear feature for which a meaningful width and orientation can be determined. Linearity is determined through correlation/regression analysis. Lead widths are measured perpendicular to the regression line, at $1 \mathrm{~km}$ 

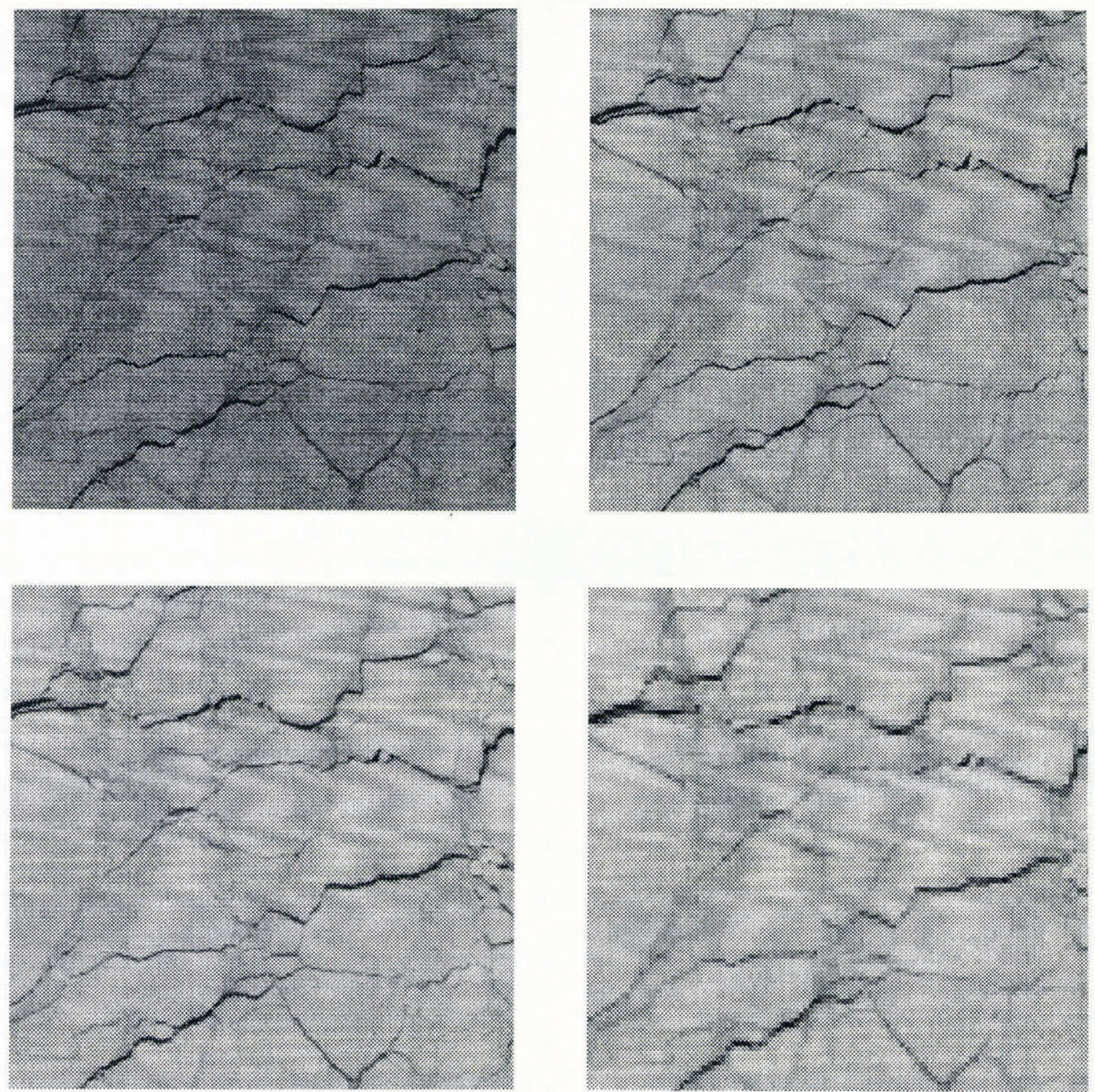

Fig. 3. Landsat MSS band 4 scene of the ice pack north of Alaska in March 1988. Area covered in the upper left image is approximately $80 \mathrm{~km}^{2}$. The remaining are degraded images with pixel sizes of 160,320 and $640 \mathrm{~m}$.
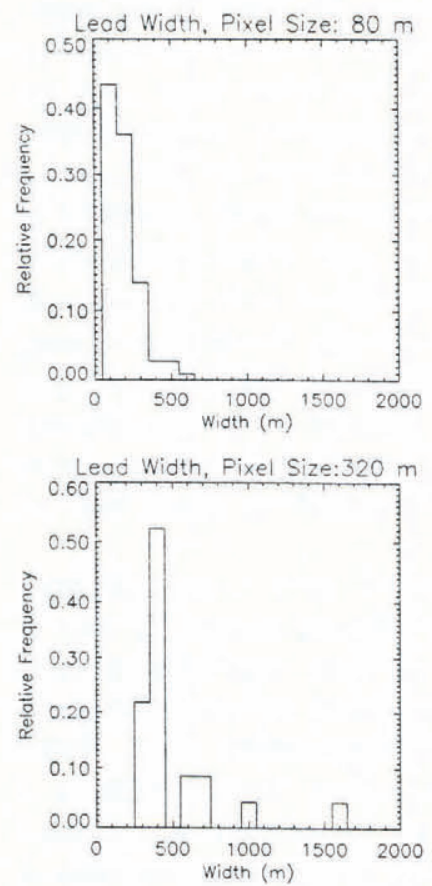
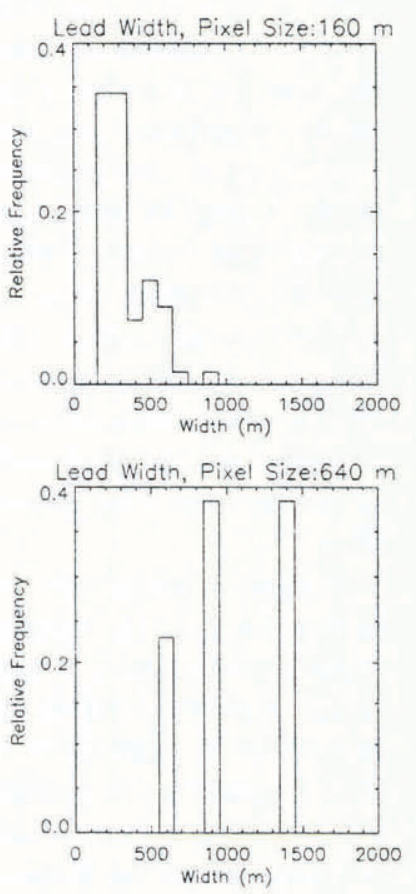

Fig. 4. Lead width distributions for the images in Figure 3. Widths are grouped in $100 \mathrm{~m}$ bins. intervals, and the slope of the regression line is the measure of the lead orientation.

One of the Landsat scenes analyzed is shown in Figure 3. Image size is $80 \times 80 \mathrm{~km}$, a sub-set of the full Landsat scene. The corresponding distribution of lead widths is shown in Figure 4. The disappearance of small leads due to a lack of contrast and the increase in the relative frequency of large leads as pixel size increases can readily be seen. In this particular image, a width threshold of approximately $250 \mathrm{~m}$ divides those leads which would disappear in the 320 and $640 \mathrm{~m}$ degradations. However, the criteria for how a given lead will "grow" or disappear during image degradation must include a measure of its thermal contrast to the surrounding ice. For example, a narrow, open lead might grow during the first degradation, accompanied by a drop in contrast due to smoothing, and then disappear in the subsequent degradation. A narrow refrozen lead, in comparison, might disappear during the first degradation. This change in lead widths as a function of pixel size affects the total 
Table 1. Changes in lead area fraction with pixel size in one Landsat scene

Pixel size Lead area fraction

Lead area

$\mathrm{m}$

$\begin{array}{rr}80 & 0.030 \\ 160 & 0.027 \\ 320 & 0.023 \\ 640 & 0.016\end{array}$
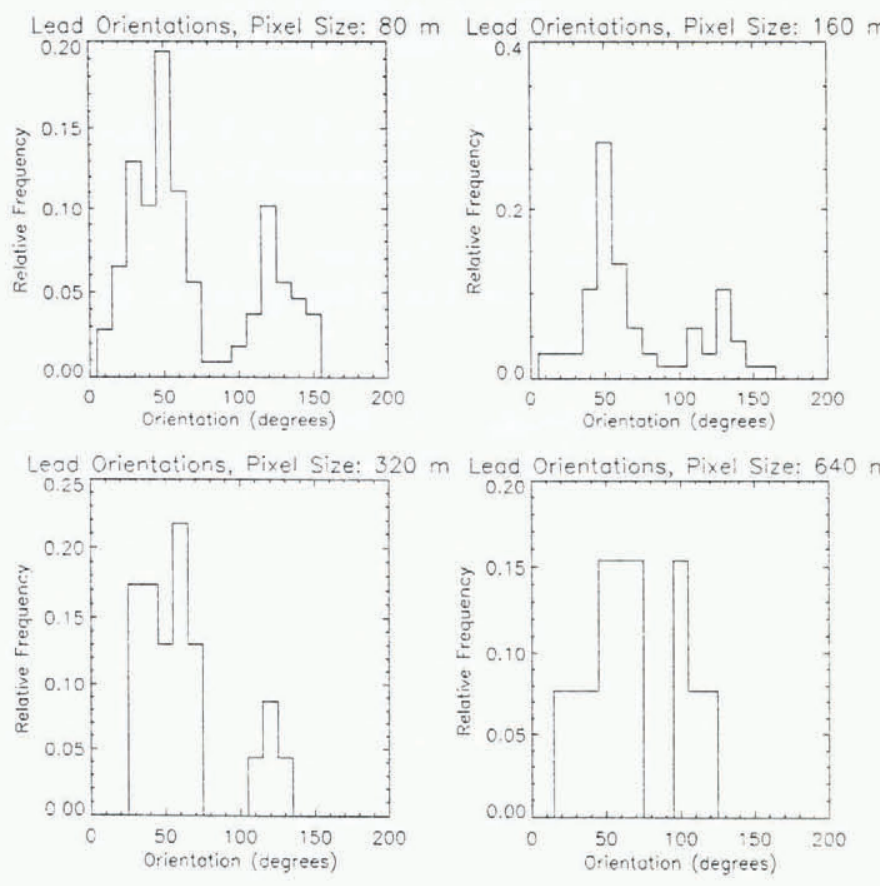

Fig. 5. Lead orientations for the degraded Landsat series shown in Figure 3. Orientation is the angle that a lead makes with the horizontal axis, measured counterclockwise.

lead area as shown in Table 1 for the images in Figure 3, where lead area fraction decreases with increasing pixel size. The distribution of lead orientation can also be expected to change, if anisotropy (i.e. a preferred orientation) exists. An illustration of this is shown in Figure 5 for the Landsat image in Figure 3. Results from other Landsat scenes show similar patterns.

Given that very small features will generally not be resolved, the issue then becomes one concerning the possibility of using the partial distribution of a particular statistic (e.g. lead widths) measured at low resolution to estimate the complete or "true" distribution of that parameter. For example, assume that lead widths, $x$, follow a negative exponential distribution with an unknown mean, $\lambda$. From a sampling point of view it is useful to treat the distribution of widths as discrete and address the number $n_{i}$ of leads in bin $i$ that have widths between $x_{i}$ and $x_{i}+w$ where $w$ is the width of the bin:

$$
n_{i}=\frac{N w}{\lambda} \mathrm{e}^{-x_{i} / \lambda}
$$

where $N$ is the unknown total number of leads in the spatial area. The idea is that $n_{i}$ is measured for a few bins, and that $\lambda$ and $N$ are estimated. To accomplish this, Equation (1) is rewritten in linear form as

$$
\ln \left(n_{i}\right)=\ln \left(\frac{N w}{\lambda}\right)-\frac{1}{\lambda} x_{i} .
$$

Letting $a=\ln (N w / \lambda)$ and $b=\lambda^{-1}$ and solving for $a$ and $b$ by the method of least squares with the observed data, the mean of the distribution and the total number of leads can then be estimated.

Experiments with this model show it to be very sensitive to the bin width and the number of bins in which leads actually occurred in the low resolution imagery. This is not unexpected considering that the entire range of $x$ is being estimated in the least squares model by observations in only one part of its entire range (such extrapolation is not recommended). The problem can be alleviated somewhat by including a few observations of small lead widths; for example, measurements from a few $\mathrm{SAR}$ images within the same area.

\section{SUMMARY AND CONCLUSIONS}

The use of the thermal channels of the AVHRR for the detection and mapping of leads has been evaluated for varying atmospheric and surface conditions. Our approach included both modeling and empirical studies, discussed with respect to lead width. Concerning atmospheric effects, the detectability of leads is dependent on the degree of thermal contrast between surface types, which varies with surface and atmospheric conditions as well as sensor response. The transfer of radiation through the atmosphere is modeled using data from Arctic ice islands and climatological values of atmospheric chemical constituents. The satellite radiances in the three AVHRR thermal channels are simulated for a variety of surface and atmospheric conditions: a range of satellite view angles, four ice thicknesses (three within leads), varying aerosol and ice crystal optical depths, and thin cirrus clouds. The change in the contrast between the lead and the surrounding ice is examined as a function of optical depth. For example, at ice crystal optical depths of more than 1.5 under mean January conditions in the central Arctic, the split-window brightness temperature differences between $2 \mathrm{~m}$ and $5 \mathrm{~cm}$ thick ice are similar to the ice temperature variability. Therefore, thresholding operations will not be able to distinguish between ice and leads. Due to the wide variety of atmospheric and surface conditions that exist in the Arctic, additional experiments in this area must be performed before general statements about the determination of optimal thresholds can be made.

Concerning measurement scale, Landsat MSS data have been successively degraded from $80 \mathrm{~m}$ to $1.2 \mathrm{~km}$ fields-of-view, using the modulation transfer function for the sensor. It can be seen that small leads disappear in the coarser resolution data and large, bright leads "grow". 
The total lead fraction decreases with resolution. Lead orientation distributions also change with increasing pixel size indicating that, in the imagery examined, small leads exhibit different orientations from larger leads. The possibility of using lead width distributions from AVHRR data to estimate the "true" lead width distribution is also assessed, where their distribution is used to predict the first moments of the true (hypothesized) negative exponential distribution. The results are shown to be very sensitive to the number and range of lead widths measured, but the accuracy of the procedure can be improved dramatically with a few samples of the same lead network at a higher resolution, e.g. from SAR data.

\section{ACKNOWLEDGEMENTS}

This work was supported by ONR grant N00014-90-J1840 and NASA grant NAGW-2407 (University of Washington subcontract \#721566).

\section{REFERENCES}

Curry, J. A., F. G. Meyer, L. F. Radke, C. A. Brock and E. Ebert. 1990. Occurrence and characteristics of lower tropospheric ice crystal in the Arctic. Int. 7. Climatol., $\mathbf{1 0}(7), 749-764$.
Eppler, D. T. and W. E. Full. In press. Polynomial trend surface analysis applied to AVHRR images to improve definition of Arctic leads. Remote Sensing Environ.

Justice, C. O., B. L. Markham, J. R. G. Townshend and R. L. Kennard. 1989. Spatial degradation of satellite data. Int. 7. Remote Sensing, 10(9), 1539-1561.

Kneizys, F.X. and 7 others. 1988. Users' guide to LOWTRAN 7. Environmental Research Papers 1010. (AFGL-TR-88-0177.)

Maykut, G. A. 1982. Large-scale heat exchange and ice production in the central Arctic. 7. Geophys. Res., 87(C10), 7971-7984.

Shenk, W. E. and V. V. Salomonson. 1972. A simulation study exploring the effects of sensor spatial resolution on estimates of cloud cover from satellites. 7. Appl. Meteorol., 11, 214-220.

Townshend, J.R. G. and C. O. Justice. 1988. Selecting the spatial resolution of satellite sensors required for global monitoring of land transformations. Int. J. Remote Sensing, 9(2), 187-236.

Wielicki, B. A. and R. M. Welch. 1986. Cumulus cloud properties derived using Landsat satellite data. J. Appl. Meteorol., 25, 261-276.

Woodcock, C. E. and A. H. Strahler. 1987. The factor of scale in remote sensing. Remote Sensing Environ., 21, $311-$ 316.

The accuracy of references in the text and in this list is the responsibility of the authors, to whom queries should be addressed. 